\title{
Montesano Montessori, Nicolina, Michael Farrelly, und Jane Mulderrig (eds.) (2019): Critical Policy Discourse Analysis. Advances in Critical Policy Studies
}

Cheltenham/Northampton: Edward Elgar Publishing. 296 Seiten. $\$ 145$

\section{Sybille Münch}

Online publiziert: 2. November 2020

(C) Der/die Autor(en) 2020

Hinter der Begriffsneuschöpfung „Critical Policy Discourse Analysis“ verbirgt sich der Versuch der HerausgeberInnen des vorliegenden Sammelbandes, die theoretischen und methodologischen Synergien einer Verbindung von kritischer PolicyForschung und dem Programm der Critical Discourse Analysis aufzuzeigen. Ein lobenswertes, wenn auch ehrgeiziges Unterfangen: Hier werden nämlich zwei Strömungen verbunden, die schon in sich durch ein hohes Maß an theoretischer Komplexität, konzeptioneller Dichte und vor allem auch Heterogenität gekennzeichnet sind.

Critical Policy Studies (CPS) zeichnen sich durch die enge Verbindung von Politischer Theorie und Policy-Analyse aus und sind aus den Debatten infolge der ,argumentativen“ oder ,interpretativen“ Wende hervorgegangen. Ihre VertreterInnen nehmen sprachlich vermittelte und geprägte Interpretationsprozesse und Interpretationskämpfe im Zuge der gesellschaftlich verbindlichen Problembearbeitung in den Blick. Diese im weitesten Sinne sozialkonstruktivistische oder post-positivistische Forschung firmiert oftmals auch als ,interpretative Policy-Analyse“, wobei diese Sammelbezeichnung mit einer Reihe von Inkonsistenzen verbunden war und in jüngerer Zeit zunehmend von der Selbstbezeichnung CPS abgelöst wird. Denn zum einen versammelten sich unter dem Oberbegriff der interpretativen Policy-Analyse sowohl solche ForscherInnen, die einer interpretativ-hermeneutischen Strömung zuzuordnen sind - die also die Deutungsleistungen von handelnden und interagierenden Akteuren in den Mittelpunkt stellen -, als auch poststrukturalistische AutorInnen, die in Anlehnung an Foucault beleuchten, wie Subjekte überhaupt erst durch den Diskurs konstituiert werden. Zum anderen wurde der interpretative Zugang als eine notwendige, nicht aber hinreichende Bedingung für Kritik erachtet. Interpretative

S. Münch (凶)

Zentrum für Demokratieforschung, Leuphana Universität Lüneburg, Lüneburg, Deutschland

E-Mail: sybille.muench@1euphana.de 
Ansätze zerstörten den dogmatischen Kern der traditionellen Interpretation nicht, so der Vorwurf in Rückgriff auf Habermas, sie klärten sie nur. Ein hermeneutisches Verstehen müsse durch kritische Reflexion ergänzt werden, wenn es Perspektiven für eine emanzipatorische Praxis eröffnen soll.

Die Kritische Diskursanalyse wiederum bzw. genauer die Critical Discourse Analysis (CDA) hat ihre Wurzeln eher in der Linguistik als in der Policy-Forschung. Ihre VertreterInnen, unter ihnen vor allem Fairclough und Wodak, haben sich jedoch wiederholt zu policy-analytischen Fragestellungen verhalten und werden vor allem von den AdvokatInnen einer poststrukturalistischen Policy-Analyse der Essex School sowie den VerfechterInnen einer Cultural Political Economy (CPE) nach Jessop und Sum rezipiert. Dementsprechend sind es gerade diese Strömungen der CPS, die im Sammelband aufgegriffen werden. Überhaupt handelt es sich bei der CDA um ein sehr heterogenes, interdisziplinäres Forschungsprogramm. Das gemeinsame Interesse besteht laut Wodak darin, Ideologien und Macht durch die systematische Analyse semiotischer Daten zu entmystifizieren. Die CDA arbeitet deutlich stärker mit linguistischen Kategorien als andere Diskurstheorien und rekonstruiert Diskurse weniger auf der Makroebene als anhand detaillierter Textanalysen.

Mit dem Entwurf einer „Critical Policy Discourse Analysis“ verbinden die HerausgeberInnen drei Anliegen: Den Nachweis einer konzeptionell reicheren Analyse von Policies, eine explizite Offenlegung der methodologischen Schritte sowie die Reflektion, inwiefern eine Integration der beiden Zugänge beide Seiten befruchten kann. Anhand von zehn Fallstudien, die durch eine konzeptionelle Einleitung und eine Schlussbetrachtung durch die HerausgeberInnen eingebettet sind, sollen diese Ziele erreicht werden. Jedes Kapitel - und hierin besteht der Mehrwert des Sammelbandes - führt sehr transparent durch die methodologischen und forschungspraktischen Entscheidungen und inwiefern diese durch die theoretische Rahmung motiviert sind.

Lesenswert ist zunächst die Einleitung der HerausgeberInnen, die die Integration von CPS und CDA klar herausarbeiten, knapp in drei zentrale Strömungen innerhalb der CDA einführen und zentrale Begrifflichkeiten definieren. Die Fallstudien eröffnet Nicolina Montesano Montessori, die mithilfe einer Text Oriented Discourse Analysis nach Fairclough den kontrahegemonialen Diskurs der zapatistischen Bewegung in Mexiko analysiert. In ihrer überaus anschaulichen Darstellung stützt sie sich auf den gramscianischen Hegemonieansatz und führt die Diskurstheorie nach Laclau und Mouffe mit verschiedenen Strängen der CDA zusammen. In einem weiteren sehr instruktiven Kapitel bringt Mitherausgeberin Jane Mulderrig die Theorie der neoliberalen Gouvernementalität mit der CDA in einen Dialog und beleuchtet die Rolle von „Nudges“ anhand einer britischen Kampagne gegen Adipositas bei Kindern. Das Kapitel besticht durch seine gute Lesbarkeit sowie didaktische Aufbereitung und würde sich auch gut zum Einsatz in der Hochschullehre eignen.

Sarah Horrod untersucht, wie politische Reformen im englischen Hochschulwesen bestehende Strukturen, Finanzierungssysteme und sogar den Zweck der Hochschulbildung selbst infrage gestellt haben. Basierend auf dem diskurshistorischen Ansatz erhellt sie die diskursive Konstruktion von „Gemeinschaft“ und deren Verwendung zur Legitimierung von marktkonformen Lern- und Lehransätzen. In einem weiteren Beitrag zur Bildungspolitik untersucht Juan Francisco Palma Carvajal aus- 
gehend vom Konzept der Gouvernementalität in Kombination mit einem ebenfalls diskurshistorischen Ansatz, inwiefern NGOs im Rahmen der jüngsten Bildungsreform in Chile sich dem vorherrschenden neoliberalen Diskurs nicht nur nicht widersetzen, sondern sogar dazu beitrugen, die Macht des neoliberalen Modells zu verschleiern. Mikko Poutanen wiederum analysiert Aufstieg und Fall der finnischen Firma Nokia. Anhand einer Zeitungsanalyse der Jahre 2007-2013 werden konkurrierende argumentative Strukturen identifiziert, die den öffentlichen Diskurs in Finnland zugunsten einer marktfreundlichen Haltung prägten.

Mitherausgeber Michael Farrelly leistet einen Beitrag zur Frage, wie politische EntscheidungsträgerInnen soziale AkteurInnen in Texten repräsentieren. Ausgehend von Van Leeuwens methodologischer Arbeit zur CDA und der theoretischen Perspektive der CPE zeigt dieses Kapitel am Beispiel signifikanter Verschiebungen in Richtung „Wettbewerb“ in der britischen Politik zur Governance seiner Gasindustrie, wie die Repräsentation sozialer AkteurInnen analysiert werden kann. Auch dieses Kapitel lässt die Forschungspraxis gut nachvollziehen und stellt eine gelungene Verbindung von CPE und CDA her. Hier hätte sich vielleicht angeboten, das Vorgehen von Arbeiten zur ,sozialen Konstruktion von Zielgruppen“ nach Schneider und Ingram abzugrenzen, die bereits innerhalb der CPS etabliert ist. Eine weitere Integration von CPE und CDA unternehmen Hendrik Theine und Maria Rieder. Dieses Kapitel befasst sich mit der Rezeption von Pikettys Bestseller „Capital in the Twenty-First Century“ in den Printmedien vier europäischer Länder, insbesondere im Hinblick auf dessen steuerpolitische Interventionen. Neben den inhaltlich interessanten Befunden zur Routinisierung des hegemonialen Diskurses sind hier besonders die aufgedeckten Legitimationsstrategien auch für weitere Forschungsarbeiten inspirierend.

Kristof Savski demonstriert anhand einer Fallstudie zur Sprachenpolitik in Slowenien, wie die Anwendung historiografischer Methoden der Quellensammlung und ein diskursiver Ansatz eine detaillierte Beschreibung eines hochkomplexen politischen Textes ermöglichen, ohne einen direkten Zugang zu politischen Beratungen zu haben. Daniela Caterina präsentiert eine Synergie zwischen historisch-materialistischer Politikanalyse und der CDA als Instrument zur Untersuchung von Hegemoniekämpfen anlässlich einer Arbeitsmarktreform unter der Regierung von Mario Monti. Tom Bartlett untersucht Verhandlungen über Fischereirechte und die lokale Bewirtschaftung der Meeresressourcen auf den westlichen Inseln Schottlands und erörtert, wie konkurrierende Diskurse über Nachhaltigkeit von einer von der Fischerei abhängigen lokalen Gemeinschaft und nationalen und transnationalen Regierungsbehörden konstruiert werden. Das Kapitel beschreibt die sozioökonomischen und kulturellen Bedingungen hinter den konkurrierenden Diskursen und macht dabei das in der Humangeographie etablierte Konzept der räumlichen Maßstabsebene (,scale“) für kritische Policy-Forschung anschlussfähig.

Die einzelnen Beiträge arbeiten mit unterschiedlichen Ansätzen innerhalb der CPS und verbinden diese wiederum mit einer Bandbreite an methodologischen Linsen, die sie aus der CDA ableiten. Die Stärke des Sammelbandes besteht vor allem darin, die sehr feinkörnige Textanalyse der CDA für LeserInnen außerhalb der Linguistik einträglich zu machen. Der überwiegend sprachwissenschaftliche Hintergrund der AutorInnen hat indes zur Folge, dass der Brückenschlag zu den CPS in 
ihrer post-positivistischen, ,,argumentativen“ und ,interpretativen“ Tradition, der von den HerausgeberInnen in der Einleitung noch sehr klar vorgenommen wird, in den meisten Beiträgen zugunsten einer stärkeren Gewichtung von CPE, Gouvernementalitäts- und Hegemonieanalysen fehlt. Wie die HerausgeberInnen in ihrem Nachwort selbst einräumen (S. 268), tragen die hier versammelten Beiträge nicht zu denjenigen Analysen von Frames, Narrativen und Storylines bei, die sonst oft charakteristisch für kritisch-argumentative Policy-Forschung sind. Zudem wird das potenzielle erkenntnistheoretische Spannungsverhältnis zwischen den sozialkonstruktivistischen Arbeiten im Geiste einer interpretativen oder argumentativen Wende und den realistisch/materialistischen Prinzipien der CDA von den Fallstudien de-thematisiert und von den HerausgeberInnen lediglich kurz angerissen. Allein Montesano Montesso$r i$ wirft dies in ihrem individuellen Beitrag sehr klar als Herausforderung für die Integration von CPS und CDA auf.

Zugleich werden durch die Interdisziplinarität des Sammelbandes die sonst oftmals verfestigten Grenzen der politikwissenschaftlichen Teildisziplinen überwunden und es finden sich neben klassischeren Politikfeldern wie Gesundheit und Hochschulpolitik und dem üblichen Bias zugunsten europäischer Fälle auch Kapitel zu Zapatismus in Mexiko und Hegemoniekämpfen in Chile. Zudem ist es in der Vergangenheit gerade die hohe Durchlässigkeit der CPS gegenüber intellektuellen Entwicklungen in den Nachbardisziplinen sowie im Bereich der Politischen Theorie gewesen, die zur Dynamik und Kreativität kritischer Policy-Forschung beigetragen haben.

Funding Open Access funding enabled and organized by Projekt DEAL.

Open Access Dieser Artikel wird unter der Creative Commons Namensnennung 4.0 International Lizenz veröffentlicht, welche die Nutzung, Vervielfältigung, Bearbeitung, Verbreitung und Wiedergabe in jeglichem Medium und Format erlaubt, sofern Sie den/die ursprünglichen Autor(en) und die Quelle ordnungsgemäß nennen, einen Link zur Creative Commons Lizenz beifügen und angeben, ob Änderungen vorgenommen wurden.

Die in diesem Artikel enthaltenen Bilder und sonstiges Drittmaterial unterliegen ebenfalls der genannten Creative Commons Lizenz, sofern sich aus der Abbildungslegende nichts anderes ergibt. Sofern das betreffende Material nicht unter der genannten Creative Commons Lizenz steht und die betreffende Handlung nicht nach gesetzlichen Vorschriften erlaubt ist, ist für die oben aufgeführten Weiterverwendungen des Materials die Einwilligung des jeweiligen Rechteinhabers einzuholen.

Weitere Details zur Lizenz entnehmen Sie bitte der Lizenzinformation auf http://creativecommons.org/ licenses/by/4.0/deed.de. 